\title{
AMBIENTE EDUCATIVO Y PERFIL LINGÜÍSTICO COMO BASES IMPORTANTES PARA LA EDUCACIÓN INICIAL DEL NIÑO
}

Educational environment and linguistic profile as important bases for child's initial education

Ambiente educacional e perfil linguístico como bases importantes para a educação inicial da criança

\section{Bernarda Leones Araque}

Universidad San Buenaventura

\section{Mariana Larrahondo Gómez}

Universidad San Buenaventura

mariana.larrahondo@usbctg.edu.co

\author{
Nathaly Simancas Sarabia \\ Universidad San Buenaventura \\ nathalysimancas@gmail.com
}

\section{Ana Milena Torres Ortega}

Universidad San Buenaventura

ana.torres06161@gmail.com

Sandy Puello Ramos

Universidad San Buenaventura

sandypuello@hotmail.com 


\title{
Andrea Fernanda Ariza Arévalo \\ Universidad San Buenaventura andreariza28@hotmail.com \\ Glendys Yaneth López Luna \\ Universidad San Buenaventura \\ glendyslop@gmail.com
}

\begin{abstract}
Resumen
Objetivo: determinar el ambiente educativo y el perfil lingüístico de los niños durante el periodo de educación inicial en los Centros de Desarrollo Infantil Modalidad Institucional en Turbaco y Arjona.

Método: estudio cuantitativo, observacional y descriptivo; como instrumentos para la recolección de los datos se utilizó la Escala de Calificación del Ambiente de la Infancia Temprana ECERS-R, compuesta por 7 áreas o dimensiones y para la evaluación del perfil lingüístico se utilizó el inventario de desarrollo BATELLE.
\end{abstract}

Resultados: la muestra estuvo conformada por 211 niños, las edades más frecuentes en la población fueron las comprendidas entre los 49 a 59 meses con un 53\%, la edad de 36 a 48 meses con un $44 \%$ y un $3 \%$ las edades entre 60 a 71 meses; $(M=50 ; D T=7,5)$. Se evidencio un ambiente educativo bueno que permite que los niños alcancen un perfil lingüístico acorde a la edad; se observó que un 38\% de estos, logro un nivel medio y un $20 \%$ de los niños estuvo en un nivel bajo. Conclusiones: la educación inicial es una oportunidad que se les debe brindar a todos los niños y niñas, permitiendo adquirir nuevos 
conocimientos, los Centros de Desarrollo Infantil son los encargados de que esta educación inicial se logre con éxito, estos tienen lugares lúdicos donde el niño puede correr, tocar, preguntar e interactuar con otros, desarrollando habilidades y destrezas para cuando inicien su etapa escolar.

Palabras clave: ambiente educativo; lenguaje; aprendizaje; infancia; educación

\section{Abstract}

Objective: to determine the educational environment and the linguistic profile of children during the period of initial education in the Centers for Child Development Institutional Modality in Turbaco and Arjona.

Method: quantitative, observational and descriptive study; As instruments for data collection, the ECERS-R Early Childhood Environment Rating Scale was used, consisting of 7 areas or dimensions, and the BATELLE development inventory was used to assess the linguistic profile.

Results: the sample consisted of 211 children, the most frequent ages in the population were those between 49 and 59 months with 53\%, the age of 36 to 48 months with $44 \%$ and $3 \%$ ages between 60 at 71 months; $(M=50$; DT = 7.5). A good educational environment was evidenced that allows children to reach a linguistic profile according to age; It was observed that $38 \%$ of these achieved a medium level and $20 \%$ of the children were at a low level. 
Conclusions: initial education is an opportunity that must be provided to all children, allowing them to acquire new knowledge, the Child Development Centers are responsible for this initial education is achieved successfully, these have fun places where the child You can run, play, ask and interact with others, developing skills and abilities for when you begin your school stage.

Key words: language: learning; childhood; education; educational environment

\section{Resumo}

Objetivo: determinar o ambiente educacional e o perfil linguístico das crianças durante o período da educação inicial nos Centros de Modalidade Institucional para o Desenvolvimento Infantil em Turbaco e Arjona.

Método: estudo quantitativo, observacional e descritivo; Como instrumentos para a coleta de dados, foi utilizada a Escala de Avaliação Ambiental da Primeira Infância ECERS-R, que consiste em 7 áreas ou dimensões, e o inventário de desenvolvimento BATELLE para avaliar o perfil lingüístico.

Resultados: a amostra foi composta por 211 crianças, as idades mais frequentes na população foram entre 49 e 59 meses com 53\%, idade de 36 a 48 meses com $44 \%$ e $3 \%$ entre 60 e 71 meses; $(M=50 ; T D=$ 7,5). Evidenciou-se um bom ambiente educacional que permite às crianças alcançar um perfil lingüístico de acordo com a idade; Observou-se que $38 \%$ atingiram um nível médio e $20 \%$ das crianças estavam em um nível baixo. Conclusões: a educação inicial é uma 
oportunidade que deve ser oferecida a todas as crianças, permitindo que adquiram novos conhecimentos; os Centros de Desenvolvimento Infantil são responsáveis por que essa educação inicial seja alcançada com sucesso; esses são lugares divertidos onde a criança pode correr, brincar, pergunte e interaja com outras pessoas, desenvolvendo habilidades e habilidades para quando você iniciar sua fase escolar. Palavras chave: linguagem: aprendizagem; infância educação; ambiente educacional

\section{Introducción}

El Ministerio de Educación Nacional en Colombia, plantea que la educación debe ser una oportunidad que se brinda a todos los niños, es decir, educación inicial en el marco de la atención integral a lo largo de la vida, donde estos aprenden a convivir, estableciendo vínculos afectivos con pares y adultos, diferentes a los de su familia; en medio de espacios apropiados para relacionarse con el ambiente natural, social y cultural.

De acuerdo con lo anterior la Declaración Mundial sobre Educación para Todos, la educación inicial comienza desde el mismo momento del nacimiento; la familia, la comunidad y las instituciones son agentes responsables de la misma; y los entornos o ambientes son determinantes para que sea una realidad, siendo estos los ámbitos espaciales, temporales y relacionales en los cuales cada niña o niño desarrolla sus propias experiencias de vida. De igual manera, los ambientes hacen referencia a los sujetos que intervienen, a las relaciones que allí se tejen y a la manera como se apropian del aprendizaje. 
En los Centros de Desarrollo Infantil (CDI) Modalidad Institucional, existen ambientes educativos, para el aprendizaje inicial, donde interactúan diferentes elementos físicos, sensoriales, como la luz, el color, el sonido, el espacio, el mobiliario; siendo estos los espacios en los que está permitido tocar, explorar, correr, jugar, preguntar y sentir, los cuales deben contar con las condiciones que enriquezcan y potencien el desarrollo integral, además los centros deben contar con un grupo de profesionales capacitados que aporten al proceso de aprendizaje de los niños.

La educación inicial pretende promover en el niño su autonomía y autoconocimiento, facilitar el desarrollo de la confianza en sí mismos, a ser cuidados y cuidar a los demás, para sentirse seguros, partícipes, escuchados y reconocidos; paralelamente propicia el aprendizaje del mundo que nos rodea, enriqueciendo el lenguaje, las formas de expresión, los hábitos de vida saludable, y contribuyendo a la identidad de la familia, la comunidad, la cultura, el territorio y el país.

Durante este periodo, el niño descubre diversas maneras de disfrutar experiencias por medio del juego, arte, literatura y exploración del entorno, estas actividades implican modificar el medio físico, los recursos y materiales con los que trabaja y facilita la articulación de los integrantes de la comunidad educativa: padres, docentes, directivos, terapeutas y comunidad en general. En este orden de ideas, para, Abuchaim, B. Bhering, E. \& Gimenes, N. (2014), potencializar estos espacios, concibe abiertamente el desarrollo de habilidades cognitivas, del lenguaje y sociales que buscan finalmente establecer entornos de aprendizaje participativo, y la participación continua de 
profesionales que propicien el desarrollo integral y las interacciones en la cotidianidad.

De este modo, resulta importante destacar el rol del fonoaudiólogo, que tiene como objeto de estudio la comunicación humana y sus desordenes, desde las áreas de desarrollo lenguaje, cognición habla y audición; las cuales están inmersas en la educación inicial desde la estimulación temprana. El profesional en esta disciplina se constituye como uno de los actores que trabaja con un grupo interdisciplinario, con el fin de realizar actividades con padres, personal docente y niños, promoviendo dentro de los Centros de Desarrollo Infantil actividades dinámicas y lúdicas que fomenten el lenguaje, el conocimiento, la libertad, vivencias culturales y el desarrollo de la cognición en los niños.

Además, el ambiente educativo permite la interacción y el intercambio comunicativo de al menos dos actores, frente a la experiencia que viven, lo que aprenden y lo que, en este orden de ideas, el desarrollo del lenguaje se concibe como uno de los procesos fundamentales del desarrollo humano especialmente durante la infancia, donde se generan cambios de manera continua y se evidencia progreso en la creación de nuevas palabras y la utilización de acuerdo al entorno. A través de este el niño adquiere la capacidad de relacionarse en su contexto y expresar sus deseos y necesidades de forma precisa.

De acuerdo con la Asociación Americana del Habla, Lenguaje y Audición (ASHA), el lenguaje es fundamental para el desarrollo integral del niño, porque está conformado por reglas sociales que cuentan con un significado, creación y combinación de nuevas 
palabras y facilitan la integración de la realidad. Según la Organización Mundial de la Salud (OMS), debe otorgarse un especial cuidado a la evolución del lenguaje en el niño dado que su adquisición y dominio contribuyen en diferentes aspectos del desarrollo cognitivo, social y de habilidades lingüística.

Como resultado de este proceso dinámico surge el desarrollo del lenguaje receptivo como expresivo, medio fundamental de la comunicación entre los humanos lo que va a permitir a los niños y niñas expresarse, comunicarse, regular la conducta de los demás y comprender el medio que les rodea. Ambos surgen de manera paralela y se evidencian desde las primeras semanas de vida, en la adquisición de habilidades comunicativas como la intencionalidad y la intersubjetividad y en el transcurso simbólico de comunicación humana, la formulación y el pensamiento que va a permitir la interacción con sus semejantes, por medio del contenido creativo lingüístico.

En este orden de ideas, el lenguaje expresivo se produce a través de la comprensión de intercambio comunicativo y de la participación activa en ambientes de interacción.

Por lo tanto, el lenguaje expresivo no se puede dar de forma aislada, debe existir una correlación entre los componentes del lenguaje. De igual manera el lenguaje comprensivo hace referencia a la capacidad de interpretar los diferentes estímulos que se reciben, sean internos o externos; está relacionado a la interpretación de imágenes, palabras, expresiones, frases, actos y enunciados. 
Se consideró necesario identificar el nivel de desarrollo del lenguaje expresivo y receptivo en los niños, teniendo en cuenta el contexto y las personas que intervienen en este proceso. Uno de ese contexto son los Centros de Desarrollo Infantil (CDI) considerados como aquellos espacios que cuentan situaciones estructuradas para poder llevar a cabo tareas de manera intencionada, donde niños y niñas encuentran formas de comunicación diferentes a las de su hogar; y reciben estímulos e información básica que les permite aprender habilidades como, toma de turnos en la conversación, seguir con atención lo que dicen los demás, ampliar su vocabulario y expresar sus ideas en diferentes situaciones.

\section{Entendiendo la educación inicial}

La educación en la primera infancia ha de ser entendida como la base e inicio para un desarrollo físico y psicológico armonioso, para la formación de las facultades intelectuales y el desarrollo de la personalidad. En cuanto a sus características, entendemos que la educación que se presta a los niños en sus primeros años ha de ser rigurosa, sistemática y adecuada a sus necesidades.

La educación inicial para el Ministerio de Educación Nacional (2016), es un proceso permanente, continuo, de interacciones y relaciones sociales de calidad, pertinentes y oportunas, que posibilitan a los niños potenciar sus capacidades y adquirir competencias en función de un desarrollo pleno como seres humanos y sujetos de derechos. Como tal, requiere un cuidado y acompañamiento apropiado del adulto que favorezca su crecimiento y desarrollo en ambientes de socialización sanos y seguros. 
Para la Organización de los Estados Iberoamericanos Para la Educación, la Ciencia y la Cultura (OIE) la primera infancia es el ciclo progresivo más importante de los seres humanos, pues en los primeros años de vida se crean las bases madurativas y neurológicas del desarrollo, destacando que las experiencias de los niños en sus primeros años son fundamentales para su progreso subsiguiente al complejizar habilidades y capacidades necesarias para el aprendizaje y educación inicial.

\subsection{Ambiente educativo}

En efecto, se ha considerado el ambiente educativo como elemento crucial en el aprestamiento de habilidades en el aula. Herrera (2006) considera el ambiente educativo o ambiente de aprendizaje escolar como un entorno físico y psicológico de interactividad regulada en donde confluyen personas con propósitos educativos, que promueva el aprendizaje y, por ende, el desarrollo integral de los niños y niñas.

Entender cómo se gesta el ambiente educativo es citar a Flórez (2017); Duarte (2016); Valverde (2015); Chaparro (1995), quienes resaltan que las interacciones producidas en el medio, son la organización y disposición espacial, las relaciones establecidas entre los elementos de su estructura, las pautas de comportamiento que en él se desarrollan, el tipo de relaciones que mantiene las personas con los objetos, las interacciones que se producen entre las personas, los roles que se establecen, los criterios que prevalecen y las actividades que se realizan. Durante el aprendizaje escolar prima el proceso social de la construcción de conocimiento y en ella se incorporan los docentes, padres de familia y estudiantes, estos últimos actúan como aprendices 
de las diferentes asignaturas, habilidades y destrezas que se indiquen en el plan de curso.

De igual forma Jaramillo (2012) considera que el ambiente del salón de clase es esencial para favorecer el desarrollo físico, social y cognitivo de los niños y las niñas. Resalta a su vez la importancia del desarrollo integral de las personas inmersas en el proceso educativo, el cual busca promover su integración social crítica. Sin embargo, precisa Hoyuelos (2007) la importancia de este ambiente lo determina el acomodo del mobiliario y el equipo dispuesto para las exigencias pedagógicas o funcionales, de manera que se constituya en un espacio amigable para todas las personas que lo habitan, acogedor, delicado y sensible que coopere en el crecimiento integral.

En los centros de educación infantil, el proceso educativo y las interacciones con materiales y pares, estimulan el lenguaje, las capacidades intelectuales y físicas, la competencia social, así como también el bienestar emocional. Sin embargo, se debe considerar el nivel de calidad del lugar, esto garantiza permanencia del menor y los efectos positivos en su proceso de aprendizaje (Valverde, 2015).

A pesar de ello, algunas corrientes teóricas difieren de la existencia de un ambiente valido para el proceso de aprendizaje, porque la intención en algunos casos de alcanzar objetivos individuales en la calidad y cantidad contrapone la esencia fundamental del aprendizaje basado en experiencias y procesos de interacción con el otro y el medio. Es decir, los ambientes de aprendizaje deberán moverse de acuerdo al modelo educativo y a las condiciones en las que se adecue un territorio. 


\subsection{Desarrollo del lenguaje y educación inicial}

El lenguaje se define como un proceso dinámico y complejo que surge a partir de la combinación del código compartido socialmente para la materialización de conceptos a través de signos arbitrario. Según Owens (2008) "el lenguaje es considerado un sistema complejo que se encuentra constituido por diferentes componentes, los cuales deben considerarse de forma autónoma aumentar su análisis y su conocimiento".

También es visto como una conducta comunicativa propiamente de los seres humanos, está ligado con el desempeño de funciones importantes a nivel cognitivo, comunicativo y social, que permiten hacer explicitas interacciones en el contexto y convertirlas en una regulación muy complejas de acción humana. García, et al (2017) La adquisición del lenguaje construye un plano positivo en la autorregulación cognitiva y comportamental del individuo.

El desarrollo del lenguaje infantil a comprenderse desde diferentes perspectivas teóricas, Jean Piaget (1956), uno de los autores más influyentes en las teorías del desarrollo infantil, afirma que el pensamiento precede al lenguaje y a través de una esquematización más avanzada permita obtener de forma rápida las nociones del lenguaje como lo son la lingüística y la semiótica, cabe destacar que el lenguaje se enfatiza en el conjunto de signos y la capacidad de representación conceptual de la lengua. Su teoría constructivista considera que los niños construyen activamente su conocimiento por medio de una reorganización de los conceptos conocidos y de los adquiridos producto de la interacción con el entorno. 
Cárdenas (2011), por su parte, considera que el infante afronta el mundo con sus propias herramientas es decir que desde el nacimiento ya existen conexiones cerebrales que facilitan y transforman el desarrollo mental y los dispositivos lógicos que hacen posible que se pueda llevar a cabo el lenguaje. Aunque el lenguaje es fundamental para el desarrollo mental y la comprensión de lo humano no se puede llevar a cabo si no existe un vínculo esencial entre lo simbólico y la interacción en el contexto.

Molina, et al (2015) considera el proceso de adquisición del lenguaje se adquiere en dos etapas; la primera, es la etapa pre-lingüística, que inicia desde el nacimiento hasta los doce meses, abriendo paso hacia un lenguaje más estructurado; se cree que durante este ciclo los niños toman conciencia de los sonidos que produce, tanto vocálicos y consonánticos, que luego tendrán una consecuencia en el contexto próximo a desarrollarse y de esta manera aprenderá a trasmitir ideas y emociones, creando vínculos entre lo que expresa y el efecto que este produce en su entorno. Y la segunda, la etapa lingüística que después del año se considera un periodo fundamental para que el niño adquiera en su totalidad el lenguaje expresivo y comprensivo, desarrollando destrezas motoras, mejor contacto y armonía en el contexto.

En la misma línea, Cotoras (2014) reflexiona que el lenguaje expresivo facilita llevar a cabo la comunicación de los niños, garantizando el aprendizaje la organización del pensamiento y la regulación del comportamiento, en la educación infantil se fortalece el lenguaje oral expresivo este inicia a partir del balbuceo hasta llegar la adquisición de expresiones más complejas esto ocurre entre los 2 y 3 
años de edad, cuando se origina la exploración del lenguaje en diferentes contextos con el fin de aumentar el nivel léxico-semántico.

Este componente del lenguaje es primordial para llevar a cabo la comunicación, gracias a él podemos expresar pensamientos de forma oral y corporal, por lo tanto, el lenguaje expresivo está relacionado de manera directa con el lenguaje verbal y no verbal. Es importante destacar que el habla es fundamental para que se produzca de manera adecuada el lenguaje expresivo, aunque se puede expresar a través de gestos, señas o palabras (Jaramillo, 2007; Cotoras, 2014).

Ahora bien, el lenguaje receptivo según Cardona, A. et al (2013), está constituido por formatos de acción que facilitan la codificación del lenguaje, es un proceso que se da a través de niveles progresivos al igual que en el lenguaje interior. Considerando al lenguaje compresivo como la capacidad de adquirir e interiorizar los conceptos partiendo de las experiencias.

El menor desarrolla la habilidad de escuchar y comprender, según Campos (2010) y Reyes, et al (2015), el lenguaje receptivo se encuentra directamente relacionada a la capacidad de reconocer palabras, frases y locuciones. Es la destreza mediante el cual se puede discriminar lo que se escucha y entender lo que se dice, ideas y pensamiento. Resulta claro entonces que el perfil lingüístico en el ambiente educativo, representa desafíos y retos que se generan desde las iniciativas propias del niño o las incorporadas por el contexto, el jardín, los educadores, entre otros.

\section{Metodología}


La metodología de la investigación es de enfoque cuantitativo, observacional y descriptivo; se utilizaron dos instrumentos para la recolección de los datos, la y para la evaluación del perfil lingüístico se utilizó el inventario de desarrollo BATELLE.

\subsection{Escala de Calificación del Ambiente de la Infancia Temprana ECERS-R:}

La Escala de Calificación del Ambiente de la Infancia Temprana ECERS-R fue creada en el año 1998 por Harms, Clifford y Cryer en Nueva York (Estados Unidos); con el objetivo de valorar la calidad de los programas de la infancia temprana.

Instituciones como Caregiver Interaction Scale (CIS), permitieron el incremento de actividades que fomentaron el desarrollo cognitivo y socio-emocional y el modelo llamado pautas de observación de actividades pedagógicas preescolares (CEDEP), estos instrumentos fueron útiles para la validación y consolidación de esta escala, ya que esta puede ser usada por directores de programas, docentes y para la evaluación de programas. Esta escala está compuesta por 7 áreas o dimensiones:

Tabla 1. Escala ESCRS-R

\begin{tabular}{|l|ll|}
\hline \multicolumn{1}{|c|}{ Dimensiones } & \multicolumn{1}{c|}{ Descripción } \\
\hline $\begin{array}{l}\text { Espacio y } \\
\text { muebles }\end{array}$ & $\bullet \begin{array}{l}\text { La organización del espacio dentro del aula. } \\
\text { La movilidad para el cuidado, juego y aprendizaje de los niños y } \\
\text { niñas. }\end{array}$ \\
& $\bullet \begin{array}{l}\text { El espacio para realizar actividades motoras gruesas y equipos } \\
\text { disponibles. } \\
\text { Considera las condiciones en que se encuentran los espacios y } \\
\text { muebles. }\end{array}$ \\
\hline
\end{tabular}




\begin{tabular}{|c|c|}
\hline $\begin{array}{l}\text { 2. Rutinas del } \\
\text { cuidado } \\
\text { personal }\end{array}$ & $\begin{array}{l}\text { - La interacción que se da en la hora de entrada, descansos, aseo y } \\
\text { salida. } \\
\text { - Las prácticas de salud y de seguridad. }\end{array}$ \\
\hline $\begin{array}{l}\text { 3. Lenguaje } \\
\text { y razonamiento }\end{array}$ & $\begin{array}{l}\text { - } \quad \text { Todo lo que estimula al niño y/o niña para comunicarse. } \\
\text { - } \quad \text { El uso del lenguaje para desarrollar habilidades de razonamiento. } \\
\text { - } \quad \text { Uso formal del lenguaje. } \\
\text { - } \quad \text { El uso de materiales de lectura. }\end{array}$ \\
\hline 4. Actividades & - La existencia, función y contenido de cada ambiente. \\
\hline 5. Interacción & $\begin{array}{l}\text { - La supervisión a los niños y niñas. } \\
\text { - } \quad \text { Las relaciones entre el personal de cuidado y los niños y niñas. } \\
\text { - } \quad \text { Los métodos de disciplina. }\end{array}$ \\
\hline $\begin{array}{l}\text { 6. Estructura del } \\
\text { programa }\end{array}$ & $\begin{array}{l}\text { - } \quad \text { El horario. } \\
\text { - La estimulación del juego libre y tiempo en grupos pequeños. }\end{array}$ \\
\hline 7. Padre y personal & $\begin{array}{l}\text { - La supervisión y evaluación del personal. } \\
\text { - Las relaciones entre los padres y el personal, cooperación entre } \\
\text { ambos. } \\
\text { - Las oportunidades de crecimiento profesional del personal. } \\
\text { - Las provisiones para necesidades personales y profesionales del } \\
\text { personal. }\end{array}$ \\
\hline
\end{tabular}

Fuente: Elaboración propia

Cada una de estas dimensiones incluye una cantidad de ítems, para un total de 43; se analiza y se valora en un rango de 1 a 7 de la siguiente manera:

- 1 o inadecuado quiere decir que estos criterios no se encuentran de forma adecuada durante la evaluación; teniendo en cuenta que los espacios son insuficientes, las aulas carecen de iluminación y ventilación; el mobiliario es básico y desorganizado, no hay prácticas de seguridad y muy pocos libros disponibles, entre otros.

- 3 o mínimo se entiende que algunos de los criterios evaluados cumplen con el objetivo establecido; es decir, se cuanta con espacio para realizar actividades de juego y aprendizaje y se utilizan materiales básicos que estimulen los procesos. 
- 5 o bueno aplica cuando se cumplen todos los criterios evaluados, y se observa que los espacios son apropiados, accesibles, iluminados, aseados y organizados, con materiales de apoyo, para que todos los niños puedan disponer de estos y enriquecer la experiencia del aprendizaje significativo.

- 7 o excelente hace referencia a que los criterios evaluados se encuentran de forma sobresaliente a lo esperado; lo que quiere decir que los espacios son lo suficientemente amplios, contando el sitio con diferentes zonas dispuestas para el aprendizaje, con la organización y estructura adecuada, el personal apropiado, materiales para todos los niños, incluyendo aquellos que se encuentran en situación de discapacidad.

La implementación de esta escala tomo aproximadamente de 45 minutos, al finalizar la evaluación las puntuaciones obtenidas se sumaron suministrando una valoración de cada área.

\subsection{Inventario de desarrollo BATTELLE:}

El Inventario de desarrollo BATTELLE es un instrumento que permite la evaluación de habilidades en desarrollo en diferentes áreas que son la personal/social, conducta adaptativa, cognición, motriz y comunicación, está orientado para niños de 0 a 8 años de edad, uno de sus objetivos es valorar la evolución del niño sin dificultades y detectar a los que presentan alguna discapacidad en alguna etapa del desarrollo.

Facilitando información acerca de los niños en los aspectos fuertes y débiles en las distintas áreas, para facilitar la elaboración de un plan 
de intervención individual. Fue creado por J. Newborg, J. R. Stock y L. Wnek y está publicado por TEA ediciones en 1998. De la cruz, M, (1996).

De acuerdo a ello se inició el diseño del BATELLE, un instrumento para evaluar el progreso del niño, capaz de proporcionar una información que sirviera de base para juzgar la efectividad de programas individuales o proyectos dirigidos a niños con el mismo tipo de problemas y también como análisis de una planificación general.

La aplicación experimental de este instrumento se realizó a más de 500 niños con edades comprendidas entre 0 a 8 años, en el año 1980 en Ohio, para que esos datos pudieran utilizarse en la elaboración final del test y para calcular la fiabilidad y validez.

En México en el año 2013, se ejecutó un estudio cuyo objetivo fue determinar la validación del instrumento para la detección oportuna de problemas de desarrollo en menores de 5 años, utilizando como instrumento referencial el BATTELLE.

\subsection{Población y muestra}

Participaron del estudio 4 Centros de Desarrollo Infantil- modalidad institucional, en los Municipios de Arjona y Turbaco. Se realizó un muestreo estratificado con afijación proporcional, a través del cual se estableció el número de niños tomados en cada uno de los Centros de Desarrollo Infantil.

Para obtener el muestreo se tuvieron en cuenta los siguientes datos, población 631 niños, entre 3 y 5 años, la proporción esperada 0,4 
margen de error absoluto del 4\%, y nivel de confianza 95\%. Teniendo en cuenta lo anterior la unidad de análisis estuvo conformada por 211 niños.

\section{Resultados y discusión}

$\mathrm{Al}$ evaluar el ambiente educativo y el lenguaje expresivo y receptivo en los niños de 3 a 5 años que asisten a los centros de Desarrollo Infantil Modalidad Institucional de Turbaco y Arjona, mediante la implementación de la Escala de Calificación del Ambiente de la Infancia Temprana ECERS-R y el Inventario de desarrollo BATTELLE, se encontraron los siguientes resultados:

Figura 1. Porcentaje según la edad de los niños

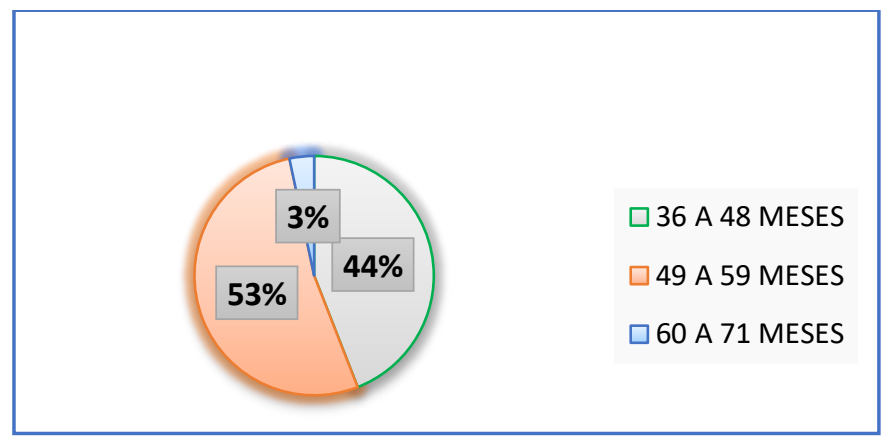

Fuente: Elaboración propia

La muestra estuvo conformada por 211 niños, las edades más frecuentes en la población fueron las comprendidas entre los 49 a 59 meses con un 53\%, la edad de 36 a 48 meses con un $44 \%$ y un $3 \%$ las edades entre 60 a 71 meses; $(M=50 ; D T=7,5)$ 
Figura 2. Porcentaje según genero de los niños

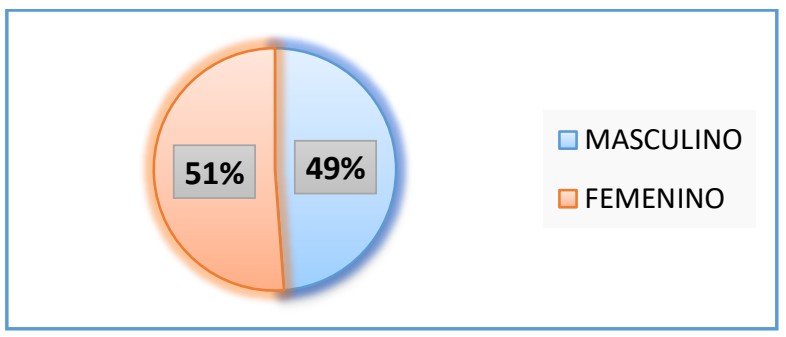

Fuente: Elaboración propia

El género predominante fue el femenino con un $51 \%$ y el masculino con un $49 \%$.

Tabla 2. Descriptivos obtenidos de la variable ambiente educativos

\begin{tabular}{cccccccc}
\hline & $\begin{array}{c}\text { Espac } \\
\text { io y } \\
\text { mueb } \\
\text { les }\end{array}$ & $\begin{array}{c}\text { Rutin } \\
\text { a de } \\
\text { cuida } \\
\text { do } \\
\text { perso } \\
\text { nal }\end{array}$ & $\begin{array}{c}\text { Lenguaje } \\
\text { razonami } \\
\text { ento }\end{array}$ & $\begin{array}{c}\text { Activida } \\
\text { d y } \\
\text { participa } \\
\text { ción }\end{array}$ & $\begin{array}{c}\text { Interacc } \\
\text { ión }\end{array}$ & $\begin{array}{c}\text { Estruct } \\
\text { ura del } \\
\text { progra } \\
\text { ma }\end{array}$ & $\begin{array}{c}\text { Padre } \\
\text { s y } \\
\text { perso } \\
\text { nal }\end{array}$ \\
\hline Media & 4,53 & 6,13 & 6,25 & 5,50 & 6,30 & 5,05 & 5,83 \\
\hline $\begin{array}{c}\text { Desviac } \\
\text { ión } \\
\text { típica }\end{array}$ & 1,21 & 0,25 & 0,50 & 0,00 & 0,63 & 1,65 & 0,77 \\
\hline $\begin{array}{c}\text { Valor } \\
\text { mínimo }\end{array}$ & 3 & 6 & 5,5 & 5,5 & 5,5 & 3 & 5 \\
\hline $\begin{array}{c}\text { Valor } \\
\text { máximo }\end{array}$ & 5,5 & 6,5 & 6,5 & 5,5 & 7 & 6,7 & 6,8 \\
\hline
\end{tabular}

Fuente: Elaboración propia

En la Tabla 2, se observa el análisis descriptivo de la variable ambiente educativo, se obtuvieron buenas puntuaciones para la Media y Desviación típica, a nivel general. El ambiente educativo permite el desarrollo del aprendizaje, según Daza, J. y Becerra, W. (2015); de acuerdo con esto se observó que en los Centros de Desarrollo Infantil la interacción entre compañeros es positivas, se fomenta el respeto 
mutuo entre los niños y adultos, involucran a los niños para resolver sus conflictos, se busca la ayuda de los profesionales concernientes cuando se presenta un problema de comportamiento; se interactúa con los niños sobre ideas relacionadas por medio del juego, haciendo preguntas para ampliar su pensamiento, a su vez se da un equilibrio entre las necesidades de examinar de manera independiente de los niños y la participación del personal en el aprendizaje, ayudan a los niños y niñas a desarrollar interacciones sociales acorde a su edad.

Figura 3. Puntuaciones obtenidas en la Escala ECER-R

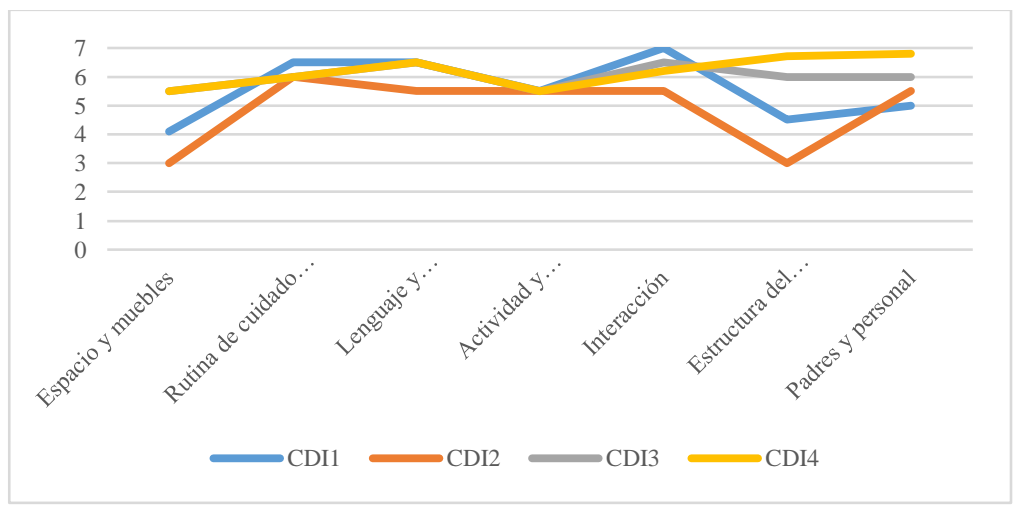

Fuente: Elaboración propia

De los cuatro Centros de Desarrollo Infantil observados se evidenció que dos de ellos alcanzaron en la variable espacio y muebles un puntaje de 3.0 y 4.1 siendo mínimo, indicando que el espacio interior es suficiente y accesible para los niños, sin embargo, se requiere que sean ampliados para que los niños puedan realizar sus actividades con mayor comodidad y la mayoría de las aulas presentan iluminación y ventilación adecuada, los espacios se encuentran limpios y bien mantenidos. De igual forma se observó que dos de los centros de 
desarrollo alcanzaron un puntaje de 5.5 respectivamente siendo un puntaje bueno, lo que demuestra que los espacios interiores son amplios y permiten a los niños y adultos moverse libremente, observándose espacios suficientes para el mobiliario y demás objetos del lugar, hay buena ventilación, iluminación natural y temperatura.

En la dimensión lenguaje y razonamiento los centros de desarrollo alcanzaron buenos puntajes; indicando que en estos lugares tienen una buena selección de libros y cuentos con muchas imágenes coloridas, los cuales son de fácil acceso para los niños, cuentan con materiales que estimulan el lenguaje, que incluyen categorías semánticas, imágenes, laminas y diferentes rincones lúdicos, los docentes leen cuentos y animan la comunicación entre los niños y las niñas, realizan juego libre, hay espacio para que los niños se expresen (dramatizados, títeres) de manera oral y a través de dibujos, también hay material para estimular el razonamiento como juegos de igual diferente, juego de tamaño y forma, juegos de clasificación.

Se observa que dos Centros de Desarrollo obtuvieron un puntaje de 3,0 y 4,5, calificando como mínimo, ya que existen rutinas secuenciales básicas que son familiares para los niños, se realizan actividades de juego libre dentro y fuera del aula, estos están supervisados para proteger la salud y seguridad de los niños, estas actividades son realizadas en pequeños grupos o de forma individual los cuales pueden ser elegidos por los niños, el personal cuenta con asesoramiento e información disponible, existe compenetraciones entre el padre y personal para cumplir con objetivos académicos. (Figura 3) 
En dos Centros de Desarrollo Infantil se obtuvo un puntaje de 6,0 y 6,7 siendo este bueno, ya que se tiene variedad en las actividades de juego que se realiza diariamente, algunas son dirigidas por el docente y otras por los niños, cada Centro de Desarrollo cuenta con una programación diaria donde los niños realizan actividades de acuerdo a una planeación establecida, dentro de estas programaciones interactúan otros profesionales como las nutricionistas, trabajo social, fonoaudiología y los padres, Se proporcionan variados juguetes, juegos, y materiales para el juego libre, los cuales son utilizados en una parte sustancial del día, tanto dentro como fuera del aula.

La educación inicial es una oportunidad que se les debe brindar a todos los niños y niñas, permitiendo adquirir nuevos conocimientos, los Centros de Desarrollo Infantil son los encargados de que esta educación inicial se logre con éxito, estos tienen lugares lúdicos donde el niño puede correr, tocar, preguntar e interactuar con otros, desarrollando habilidades y destrezas para cuando inicien su etapa escolar.

En la Tabla 3, se observa el análisis descriptivo la Media y la Desviación típica de la variable perfil lingüístico que incluye lenguaje expresivo y receptivo; utilizando el Inventario de desarrollo BATTELLE.

Tabla 3. Descriptivos obtenidos de la variable perfil lingüístico

\begin{tabular}{ccc}
\hline & Lenguaje expresivo & Lenguaje receptivo \\
\hline Media & 37,78 & 29,47 \\
\hline Desviación típica & 9,91 & 6,07 \\
\hline Valor mínimo & 12 & 14 \\
\hline
\end{tabular}


Valor Máximo

62

47

Fuente: Elaboración propia

Tabla 4. Porcentajes y frecuencias de la variable perfil lingüístico

\begin{tabular}{lcccccc}
\hline \multirow{2}{*}{ Perfil lingüístico } & \multicolumn{3}{c}{ Alto* } & \multicolumn{2}{c}{ Medio* } & \multicolumn{2}{c}{ Bajo* } \\
\cline { 2 - 7 } & $\mathrm{n}$ & $\%$ & $\mathrm{n}$ & $\%$ & $\mathrm{n}$ & $\%$ \\
\hline $\begin{array}{c}\text { Lenguaje } \\
\text { Expresivo }\end{array}$ & 88 & 42 & 81 & 38 & 42 & 20 \\
\hline $\begin{array}{l}\text { Lenguaje } \\
\text { Receptivo }\end{array}$ & 79 & 37 & 99 & 47 & 33 & 16 \\
\hline $\begin{array}{l}\text { Alto*: Por encima de lo esperado para la edad } \\
\text { Medio*: De acuerdo a patrones normativos de la edad } \\
\text { Bajo*: Por debajo de lo esperado para la edad }\end{array}$ & & & & \\
\hline
\end{tabular}

Fuente: Elaboración propia

Los porcentajes y las frecuencias que los niños alcanzaron en la variable perfil lingüístico se observan en la Tabla 4; en las sub pruebas de lenguaje expresivo y receptivo establecidas por el Inventario de desarrollo BATTELLE. En el Lenguaje Expresivo se observó en general que un $42 \%$ de los niños alcanzaron un alto nivel, realizando sin dificultad tareas como la producción y uso de sonidos, palabras o gestos. Un 38\% de estos, logro un nivel medio evidenciando el conocimiento y la capacidad que tienen para usar reglas gramaticales sencillas al organizar expresiones y frases; y un $20 \%$ de los niños estuvo en un nivel bajo, dado que presentaban dificultades en la producción de sonidos vocálicos y consonánticos diferentes, y en la articulación de sílabas y palabras de forma ininteligible.

De acuerdo con la edad un $29 \%$ de los niños entre 36 a 48 meses presentaron un nivel expresivo bajo, estando por debajo de lo esperado para la edad, observándose en ellos dificultad en la producción de palabras o gestos y poca adquisición de vocabulario. Un 50\% de los 
niños entre 49 a 59 meses lograron un nivel alto por encima de lo esperado para la edad, evidenciando la capacidad para utilizar reglas gramaticales que permiten generar diferentes formas de palabras, como los plurales, tiempos verbales, preguntas y negaciones.

De igual forma se evidencio que un $71 \%$ de los niños de 60 a 71 meses alcanzo un alto nivel en el lenguaje expresivo, produciendo diferentes sonidos vocálicos, consonánticos y combinaciones silábicas, palabras y frases con precisión, evidenciando la utilización de reglas gramaticales. Albets y De la Peña (2016); consideran que el lenguaje expresivo es un proceso neuropsicológico que permite la comunicación de los niños, la adquisición de aprendizajes, la organización del pensamiento y la regulación del comportamiento, entre otros; y exponen que la educación infantil, debe consolidar el lenguaje oral que comienza con las primeras expresiones en forma de balbuceos hasta la adquisición de expresiones más elaboradas entre los 24 y los 48 meses de edad, cuando va aumentando progresivamente el léxico semántico y los elementos gramaticales.

Los resultados encontrados al evaluar el lenguaje receptivo indicaron que el $37 \%$ de los niños alcanzo un nivel alto por encima de lo esperado para la edad realizando tareas de discriminación y comprensión del significado de las palabras; un 47\% de los niños logro un nivel medio, de acuerdo a patrones normativos para la edad, evidenciando que reconocen y comprenden sonidos y palabras; y $16 \%$ de los niños presento un nivel bajo, presentando dificultades notorias en la comprensión de mensajes y ordenes sencillas. 
De acuerdo con la edad, el 58\% de los niños entre 36 a 48 meses alcanzaron un nivel medio, estando acordes a patrones normativos para la edad, demostrando que comprenden acciones, pensamientos e ideas; un $43 \%$ de los niños entre 49 a 59 meses logro un nivel alto, evidenciando que comprenden la información recibida a través de gestos, signos, u otros medios no verbales. Y un $17 \%$ de los niños obtuvo un nivel bajo, observándose en ellos dificultades en la capacidad para comprender diferencias entre sonidos, palabras, gestos y ordenes de complejidad creciente; y un $71 \%$ de los niños entre 60 a 71 meses lograron un nivel alto en el lenguaje receptivo, mostrando que comprenden sonidos, palabras y gestos que tienen intenciones, finalidades o contenidos determinados. Por otro lado, según Acosta, E (2018), el lenguaje receptivo permite al niño captar la información a través de los sentidos, analizarla y comprenderla; este permite el vínculo entre las palabras, frases y la evocación de significados, objetos, actos y símbolos que lo representan.

\section{Conclusiones}

Los resultados obtenidos en esta investigación permiten evidenciar que los niños que asisten a los centros de desarrollo infantil, presentan un mejor desempeño a nivel del lenguaje expresivo ya que en estos espacios se realizan más actividades enfocadas en estimular la expresión del lenguaje, con el fin de que el niño pueda manifestar sus necesidades, sentimientos y lo que ocurre alrededor, a través de canciones, poesías y cuentos.

Es necesario redimensionar los ambientes educativos como un espacio que facilita los modos interacción de la población infantil, de manera 
que los centros de desarrollo infantil sean un verdadero espacio abierto, flexible, dinámico que facilite la articulación del aprendizaje significativo en el aula.

El hecho de investigar y conocer los espacios y elementos físicos que acompañan el proceso de conocimiento en la población infantil permite innovar las prácticas docentes y constituye una respuesta necesaria al continuo cambio de las actividades asociadas al desarrollo infantil.

\section{Referencias bibliográficas}

Abuchaim, B. Bhering, E. \& Gimenes, N. (2014). Reflexões sobre a avaliação de ambientes de Educação Infantil. Reuniões da ABAVE, (7), 1-14.

Acosta, E. (2018). Estrategias lúdicas para el desarrollo de la creatividad en niños de 4 a 5 años en la unidad educativa “Augusto Nicolás Martínez". 2018. tesis de maestría. [Internet]. Disponible en: http://repositorio.uti.edu.ec/handle/123456789/885

Alberts, L. y De la Peña, C. (2016). Lenguaje expresivo en Educación Infantil: clave para la estimulación de Inteligencias Múltiples. ReiDoCrea, 5, 316-321.

Campos, L. (2010). Relación entre el desarrollo personal social y los procesos evolutivos vinculados con el aprendizaje escolar en las áreas del lenguaje y la cognición. Psicogente, 13(23). 
Cardenas, P (2011). Piaget: lenguaje, conocimiento y educación. Revista colombiana de educación, (60), 71-91.

Cardona, A. Ospina, S. Trujillo, T. Arboleda, L. \& Arango, D. (2013). Desarrollo del lenguaje comprensivo y expresivo en niños de 12 a 36 meses. Revista CES Salud Pública, 4(2), 92-105.

Chaparro, C. (1995). El ambiente educativo: condiciones para una práctica educativa innovadora. especialización en gerencia de proyectos educativos y sociales. cinde-uptc. tunja.

Cotoras, P. (2’014). Lenguaje expresivo y comprensivo: Primeras palabras en cantando aprendo. [Internet]. Disponible en: http://www.cantandoaprendo.cl/pdf/columna4.pdf

Daza, J. Becerra, W. (2015). Ambientes de aprendizaje o ambientes educativos. "Una reflexión ineludible". Revista de Investigaciones. UCM, 15(25), 144-158.

De la cruz, M, (1996). Battelle Inventario De Desarrollo, libro pag 810, Madrid.

Duarte, J. (s/f) Ambientes de Aprendizaje una Aproximación Conceptual. Revista Iberoamericana de Educación.

Flórez Romero, R., Castro Martínez, J. A., Galvis Vásquez, D. J., Acuña Beltrán, L. F., \& Zea Silva, L. A. (2017). Ambientes de aprendizaje y sus mediaciones: en el contexto educativo de Bogotá.

García, D. S., Hernández, J. Á., \& Peláez, A. D. (2017). Evaluación del programa de desarrollo cognitivo" inteligencia 
XXI". Revista INFAD de Psicología. International Journal of Developmental and Educational Psychology., 1(2), 69-80.

Harms, T. Clifford, D. Cryer. D. (1998). Escala ECERS-R Early Childhood Environmental Rating Scale- Revised version; New York. [Internet]. Disponible en: http://www.psicoeducacion.eu/psicoeducacion/ECERS-R.pdf

Herrera, L. (2006) Consideraciones para el diseño didáctico de ambientes virtuales de aprendizaje: Una propuesta basada en las funciones cognitivas del aprendizaje. Revista Iberoamericana de Educación, 38(5), 1-19.

Hoyuelos, A. (2007). La escuela, ámbito estético educativo. Territorios de la infancia. Diálogos entre la arquitectura y la pedagogía. (pp. 166- 175). Barcelona: Editorial Graó.

Jaramillo, L (2007). Planta física a nivel interno y externo. Disposición del ambiente en el aula. Universidad del Norte. Instituto de Estudios Superiores en Educación.

Ministerio de Educación Nacional. La educación en Colombia. Revisión de políticas nacionales en educación. 2016. [Internet]. Disponible en: http://www.mineducacion.gov.co/1759/articles356787_recurso_1.pdf

Molina, D. P., Garcés, C. I., Puchades, O. T., \& Llongo, E. H. (2015). La adquisición del lenguaje según el modelo innatista. $3 c$ Empresa: investigación y pensamiento crítico, 4(2), 87-107. 
Owens, R. E. (2008). Desarrollo del lenguaje, 5 Ed. Editorial: PEARSON S.A; Madrid, España.

Piaget, J. (2007). Desarrollo Cognitivo: Las Teorías de Piaget y de Vygotsky

Reyes, K. C., Castilla, L. L. G., \& Lentino, Y. P. (2015). Stimulation of expressive and receptive language in the early stage of children of 2-3 years of the degree toddler. Hexágono Pedagógico, 6(1), 111-141.

Valverde, P. (2015). Ambientes de calidad en la infancia temprana. Pensando Psicología, 11(18), 141-151. 